\title{
A theoretical study of correlation between scaled energy and earthquake magnitude based on two source displacement models
}

\author{
Jeen-Hwa Wang
}

Received: 28 October 2013/Accepted: 13 January 2014/Published online: 1 February 2014

(C) The Seismological Society of China, Institute of Geophysics, China Earthquake Administration and Springer-Verlag Berlin Heidelberg 2014

\begin{abstract}
The correlation of the scaled energy, $\hat{e}=E_{\mathrm{s}} /$ $M_{0}$, versus earthquake magnitude, $M_{\mathrm{s}}$, is studied based on two models: (1) Model 1 based on the use of the time function of the average displacements, with a $\omega^{-2}$ source spectrum, across a fault plane; and (2) Model 2 based on the use of the time function of the average displacements, with a $\omega^{-3}$ source spectrum, across a fault plane. For the second model, there are two cases: (a) As $\tau \fallingdotseq T$, where $\tau$ is the rise time and $T$ the rupture time, $\lg (\hat{e}) \sim-M_{\mathrm{s}}$; and (b) As $\tau \ll T, \lg (\hat{e}) \sim-(1 / 2) M_{\mathrm{s}}$. The second model leads to a negative value of $\hat{e}$. This means that Model 2 cannot work for studying the present problem. The results obtained from Model 1 suggest that the source model is a factor, yet not a unique one, in controlling the correlation of $\hat{e}$ versus $M_{\mathrm{s}}$.
\end{abstract}

Keywords Scaled energy - Earthquake magnitude . Seismic-wave energy $\cdot$ Seismic moment $\cdot$ Source displace model

\section{Introduction}

The seismic-wave energy, $E_{\mathrm{s}}$, and seismic moment, $M_{0}$, are two main parameters quantifying earthquakes. The scaled energy, $\hat{e}=E_{\mathrm{s}} / M_{0}$, can link dynamic properties (from $E_{\mathrm{s}}$ ) and static behavior (from $M_{0}$ ) of earthquake sources, and, thus, the correlation of $\hat{e}$ versus $M_{\mathrm{s}}$ would reflect the source properties (cf. Kanamori and Heaton 2000; Kanamori and Rivera 2004). The measured values of $\hat{e}$ range from $10^{-6}$ to $10^{-3}$

J.-H. Wang $(\bowtie)$

Institute of Earth Sciences, Academia Sinica, Nangang,

P.O. Box 1-55, Taipei, China

e-mail: jhwang@earth.sinica.edu.tw
(Kanamori 1977; Vassiliou and Kanamori 1982; Kikuchi and Fukao 1988; Choy and Boatwright 1995; Brodsky and Kanamori 2001; Hwang et al. 2001; Huang et al. 2002, 2009; Kinoshita and Ohike 2002). A large range of $\hat{e}$ might be due to the uncertainty of measuring $E_{\mathrm{s}}$, because the signals are often affected by several factors, including finite frequency bandwidth limitation as the major one (cf. Wang 2004). From the equation to express the effect due to finite frequency bandwidth limitation shown in Wang (2004), it is obvious that the under-estimate is larger for large earthquakes than for small ones. On the other hand, the estimate of $M_{0}$ from longerperiod seismic data has lower uncertainty.

Brodsky and Kanamori (2001) first claimed an abrupt rise in $\hat{e}$ from $10^{-6}$ to $10^{-4}$ almost at $M_{\mathrm{w}}=5$, where $M_{\mathrm{w}}$ is the moment magnitude, that is, $\hat{e}$ is of size-dependence. On the contrary, Ide and Beroza (2001) stated that the values of $E_{\mathrm{s}}$, especially for $M_{\mathrm{w}}<5$ events, used by Brodsky and Kanamori (2001) were underestimated due to finite frequency bandwidth limitation. They corrected the $E_{\mathrm{s}}$, especially for intermediate-sized and small events, based on a $\omega^{-2}$ source model, and then stressed that $\hat{e}$ is almost a constant of about $3 \times 10^{-5}$ in a large range of $\lg \left(M_{0}\right)$ from 4 to 21 . Kinoshita and Ohike (2002) stated that $\hat{e}$ is weakly dependent on $M_{0}$.

From a 1-D elastodynamic earthquake fault model, Shaw (1998) stressed that the $\hat{e}$ is a function of $M_{0}$ and affected by friction. Kanamori and Rivera (2004) studied size-dependence of $\hat{e}$ on $M_{\mathrm{w}}$ through the formulation of the problem using variational calculus. They related the minimum of $\hat{e}$ (denoted as $\hat{e}_{\min }$ ) to several source parameters. They stated that the $M_{0} \sim f_{\mathrm{c}}^{-3}$ scaling leads to independence of $\hat{e}_{\min }$ on $M_{\mathrm{w}}$, while the $M_{0} \sim f_{\mathrm{c}}^{-(3+\varepsilon)}$ scaling, with $0<\varepsilon \leq 1$, results in an increase in $\hat{e}_{\min }$ with $M_{\mathrm{w}}$. They also claimed that the predicted value of $\hat{e}_{\min }$ for the largest crustal earthquakes is close to the average for large events, and $\varepsilon=0.5$ is the optimum value. In order to 
minimize the functional $\mathrm{J}(u, v)=E_{\mathrm{s}}-\lambda M_{\mathrm{o}}$, where $u(t)$ is the radiation pattern-corrected far-field displacement in terms of time $t, v(t)=\mathrm{du}(t) / \mathrm{d} t$, and $\lambda$ is the Lagrangian multiplier, Kanamori and Rivera (2004) considered $u(t)$ to be a one-sided pulse with a total duration of $T$, and thus, they took $u(0)=u(T)=0$ to be the initial conditions for solving the equation of the displacement. The parameter $T$ is the source rupture duration. They obtained the velocity: $v(t)=\left(6 M_{0} / C_{\mathrm{M}} T^{3}\right)(T-2 t)$, where $C_{\mathrm{M}}$ is a factor in describing $M_{0}$ as explained below, and the displacement: $u(t)=\left(6 M_{0} / C_{\mathrm{M}} T^{3}\right) t(T-t)$. Based on $u(t)$ and $v(t)$, they obtained $\hat{e}_{\min }=12 C_{\mathrm{E}} M_{0} / C_{\mathrm{M}}^{2} T^{3}$, where $C_{\mathrm{E}}$ is a factor in describing $E_{\mathrm{s}}$ as explained below, in the extreme state of $E_{\mathrm{s}} / M_{0}$. This gives a fact that $\hat{e}_{\text {min }}$ is independent upon $M_{\mathrm{s}}$. Their results lead to a positive velocity of $6 M_{0} / C_{\mathrm{M}} T^{2}$ at $t=0$ and a negative one of $-6 M_{0} / C_{\mathrm{M}} T^{2}$ at $t=T$.

Kanamori and Rivera (2004) obtained significant results for understanding the correlation between $\hat{e}_{\min }$ and $M_{\mathrm{s}}$. Nevertheless, it is still necessary to examine the correlation when different initial conditions are used, because the sum of the waveforms generated from the two planes of a fault recorded at a remote observational point is not always to form a one-sided pulse with $u(0)=u(T)=0$ as used in Kanamori and Rivera (2004). In addition, they did not concern the type of the source spectra. The finite frequency bandwidth effect can affect the measure of seismic radiation energy (cf. Wang 2004). Under such an effect, different source spectra will lead to different measured values of seismic radiation energy, thus resulting in different estimated ones of scaled energy. Hence, it is also necessary to study dependence of $\hat{e}$ on $M_{\mathrm{s}}$ based on a $\omega^{-2}$ source model.

In this study, an attempt is made to theoretically investigate the correlation of $\hat{e}$ versus $M_{\mathrm{s}}$ using two models: (1) the use of the time function of the average displacements, with a $\omega^{-2}$ source spectrum, across a fault plane; and (2) the use of the time function of the average displacements, with a $\omega^{-3}$ source spectrum, across a fault plane. The time functions were proposed by Beresnev and Atkinson (1997). For the first way, like Kanamori and Rivera (2004) the source model is not prescribed, and only the extreme state of $E_{\mathrm{S}} / M_{0}$ is taken into account. On the other hand, for the second one, a $\omega^{-2}$ source model is considered, and it is not necessary to minimize a functional. Since the observed seismograms are quite complex, Model 1 is just a special case of the problem. Comparison of the present results and those obtained by Kanamori and Rivera (2004) will be discussed below.

\section{Theory and discussion}

Following Kanamori and Rivera (2004), from the radiation pattern-corrected far-field displacement, $u(t), E_{\mathrm{s}}$, and $M_{0}$ are defined to be:
$E_{\mathrm{s}}=C_{\mathrm{E}} v^{2}(t) \mathrm{d} t$

$M_{0}=C_{\mathrm{M}} u(t) \mathrm{d} t$.

In Eqs. (1) and (2), $v=\mathrm{d} u / \mathrm{d} t, C_{\mathrm{E}}=8 \pi \rho r^{2} \beta / 5$, and $C_{\mathrm{M}}=4 \pi \rho r \beta^{3}$, where $r$ is the hypocentral distance between an event and a recording station and $\rho$ and $\beta$ are, respectively, the density and shear-wave velocity in between the source and the observational point, and the integration is performed from 0 to $T$. The radiation pattern-corrected farfield displacement, $u(t)$, is related to the velocity, $v(t)$, which is the first derivate of the average displacement, $\mathrm{d}(t)$, across a faut plane in the form: $u(t) \sim v(t-r / \beta)$.

The far-field time functions of the displacement and velocity are commonly complex, because there are numerous factors in affecting them. At a hard rock site, without substantial site effects, the modulus of shear-wave displacement spectrum can be written as

$D(\omega)=2 S(\omega) P(\omega) \exp (-\omega r / 2 Q \beta)$,

where $\omega$ is the angular frequency, $Q$ is the quality factor, $S(\omega)$ is the source spectrum, $P(\omega)$ accounts for the spectral cutoff above a certain frequency. $S(\omega)$ is modeled by multiplying a deterministic function by the Fourier spectrum of windowed Gaussian noise (cf. Beresnev and Atkinson 1997). There are different ways to select the deterministic function, for example, the $\omega^{-2}$ or $\omega^{-3}$ model (e.g., Aki 1967; Brune 1970). In this study, we apply the source time functions proposed by Beresnev and Atkinson (1997). An expression for the displacement from a point shear dislocation in a homogeneous elastic space (Aki and Richards 1980), together with a definition of the seismic moment, $M(t)=\mu u_{\mathrm{o}}(t) A$, where $\mu, u_{\mathrm{o}}(t)$, and $A$ are the rigidity, time function of source displacement, and fault area, gives the far-field shear wave in the form

$u(x, t)=\left(R_{\theta \phi} / 4 \pi r \beta^{3} r\right) v_{\mathrm{o}}(t-r / \beta)$,

where $u(x, t)$ is the displacement at spatial point $x, m$ is the shear modulus, $R_{\theta \phi}$ is the angular radiation pattern, $v_{\mathrm{o}}(t)$ is the time derivative of the average displacement across the fault plane, and $A$ is the fault area.

The studies of the correlation of $\hat{e}$ versus $M_{\mathrm{s}}$ using two models as mentioned above are described below.

\subsection{Model 1}

This model is based on the condition that the average source displacement, $d(t)$, shows a $\omega^{-2}$ source spectrum as proposed by Aki (1967). Beresnev and Atkinson (1997) proposed three time functions of the displacements across a fault plane, which start from zero and approach a certain level $D_{\mathrm{o}}$, to satisfy the request that the spectra are a $\omega^{-n}$ form. In this study, we apply the time functions of the 
source displacements with a $\omega^{-2}$ spectrum as described by the following expression:

$d_{2}(t)=D_{\mathrm{o}}\left[1-(1+t / \tau) \mathrm{e}^{-t / \tau}\right]$,

where $\tau=1 / \omega_{\mathrm{c}}, \tau$ is the rise time, $\omega_{\mathrm{c}}=f_{\mathrm{c}} / 2 \pi$, and $D_{\mathrm{o}}=d_{2}(t \rightarrow \infty)$. The modulus of the Fourier transform of $d_{2}(t)$ is $D_{2}(\omega)=D_{\mathrm{o}} /\left[1+\left(\omega / \omega_{\mathrm{c}}\right)^{2}\right]$, which is a $\omega^{-2}$-form function at high frequencies. The time function of the source velocity is:

$v_{2}(t)=D_{0}(t / \tau) \mathrm{e}^{-t / \tau} / \tau$.

Inserting Eqs (4) and (6), respectively, into Eqs (1) and (2) gives

$E_{\mathrm{s}}=\left(C_{\mathrm{E}} D_{\mathrm{o}}^{2} / \tau\right)\left\{1-\left[1+2 T / \tau+2(T / \tau)^{2}\right] \mathrm{e}^{-2 T / \tau}\right\} ;$

$M_{\mathrm{o}}=\left(C_{\mathrm{M}} D_{\mathrm{o}} \tau\right)\left\{\left(1+\mathrm{e}^{-T / \tau}\right)(T / \tau)+2 \mathrm{e}^{-T / \tau}-2\right\}$.

where $\eta=T / \tau$. Dividing Eq (7) by Eq (8) gives

$$
\begin{aligned}
\hat{e}= & C_{\mathrm{E}} D_{\mathrm{o}}\left\{1-\left[1+2 T / \tau+2(T / \tau)^{2}\right] \mathrm{e}^{-2 T / \tau}\right\} / \\
& C_{\mathrm{M}} \tau^{2}\left\{\left(1+\mathrm{e}^{-T / \tau}\right)(T / \tau)+2 \mathrm{e}^{-T / \tau}-2\right\} .
\end{aligned}
$$

Eq (9) shows that $\hat{e}$ is a complex function of $T / \tau$. In principle, for normal earthquakes $\tau$ cannot be larger than $T$ (cf. Kanamori and Anderson 1975). There are two assumptions of relating $\tau$ to $T$ : (1) $\tau \fallingdotseq T$ (from a conventional viewpoint); and (2) $\tau \ll T$, which is from the slip-pulse concept proposed by Heaton (1990). As $T / \tau \fallingdotseq 1, \hat{e} \fallingdotseq 3.12\left(C_{\mathrm{E}} D_{\mathrm{o}} / C_{\mathrm{M}} \tau^{2}\right)$, thus, giving $\hat{e} \sim \tau^{-2} \sim f_{\mathrm{c}}^{2}$. Since $f_{\mathrm{c}} \sim M_{0}^{-1 / 3}$ (Brune 1970), we have $\hat{e} \sim M_{0}^{-2 / 3}$ or $\lg (\hat{e}) \sim-(2 / 3) \lg \left(M_{0}\right)$. This leads to $\lg (\hat{e}) \sim-M_{\mathrm{s}}$ as considering the PB relationship of $M_{0}$ versus $M_{\mathrm{s}}$ obtained by Purcaru and Berckhemer (1978). Obviously, $\hat{e}$ negatively correlates to $M_{\mathrm{s}}$, with a scaling exponent of -1 .

As $\tau \ll T, 1-\left[1+2 T / \tau+2(T / \tau)^{2}\right] \mathrm{e}^{-2 T / \tau} \fallingdotseq 1$ and $(T / \tau)$ $\left(1+\mathrm{e}^{-\mathrm{T} / \tau}\right)+2 \mathrm{e}^{-\mathrm{T} / \tau}-2 \fallingdotseq T / \tau$. Hence, we have $\hat{e} \fallingdotseq C_{\mathrm{E}} V /$ $C_{\mathrm{M}} \tau$, where $V=D_{\mathrm{o}} / T$ is the average particle velocity. Obviously, this gives $\hat{e} \sim \tau^{-1} \sim f_{\mathrm{c}}$. Since $f_{\mathrm{c}} \sim M_{0}^{-1 / 3}$ (Brune 1970), we have $\hat{e} \sim M_{0}^{-1 / 3}$ or $\lg (\hat{e}) \sim-(1 / 3) \lg \left(M_{0}\right)$. This leads to $\lg (\hat{e}) \sim-(1 / 2) M_{\mathrm{s}}$ as considering the PB relationship. Obviously, $\hat{e}$ negatively correlates to $M_{\mathrm{s}}$, with a scaling exponent of $-1 / 2$.

\subsection{Model 2}

This model is based on the condition that the average source displacement, $d(t)$, shows a $\omega^{-3}$ source spectrum as first proposed by Haskell (1966), and then deeply studied by Aki (1967). Beresnev and Atkinson (1997) proposed three time functions of the displacements across a fault plane, which start from zero and approach a certain level $D_{\mathrm{o}}$, to satisfy the request that the spectra are a $\omega^{-\mathrm{n}}$ form. In this study, we apply the time function of the source displacements with a $\omega^{-3}$ spectrum as described by the following expression:

$d_{3}(t)=D_{\mathrm{o}}\left\{2-\left[1+(1+t / \tau)^{2}\right] \mathrm{e}^{-t / \tau}\right\}$,

where $D_{\mathrm{o}}=d_{3}(t \rightarrow \infty)=d_{2}(t \rightarrow \infty)$. The modulus of the Fourier transform of $d_{3}(t)$ is $D_{3}(\omega)=D_{\mathrm{o}} /[1+(\omega /$ $\left.\left.\omega_{\mathrm{c}}\right)^{2}\right]^{3 / 2}$, which is a $\omega^{-3}$-form function at high frequencies. The time function of the source velocity is:

$v_{3}(t)=D_{\mathrm{o}}(t / \tau)^{2} \mathrm{e}^{-t / \tau} / \tau$.

Inserting Eqs (11) and Eq. (10), respectively, into Eqs (1) and (2) gives

$$
\begin{aligned}
E_{\mathrm{s}}= & \left(C_{\mathrm{E}} D_{\mathrm{o}}^{2} / 4 \tau\right)\left\{3-\mathrm{e}^{-2 T / \tau}\left[3-6(T / \tau)-6(T / \tau)^{2}\right.\right. \\
& \left.\left.-4(T / \tau)^{3}+2(T / \tau)^{4}\right]\right\} ; \\
M_{\mathrm{o}}= & \left(C_{\mathrm{M}} D_{\mathrm{o}} \tau\right)\left[6-2(T / \tau)-6 \mathrm{e}^{-T / \tau}-4(T / \tau) \mathrm{e}^{-T / \tau}\right. \\
& -2(T / \tau)^{2} \mathrm{e}^{-T / \tau} .
\end{aligned}
$$

Dividing Eqs (12) by (13) gives

$$
\begin{aligned}
\hat{e}=( & \left.C_{\mathrm{E}} D_{\mathrm{o}} / 4 C_{\mathrm{M}} \tau^{2}\right)\left\{3-\mathrm{e}^{-2 T / \tau}\left[3-6(T / \tau)-6(T / \tau)^{2}\right.\right. \\
& \left.\left.-4(T / \tau)^{3}+2(T / \tau)^{4}\right]\right\} /[6-2(T / \tau) \\
& \left.-6 \mathrm{e}^{-T / \tau} 4(T / \tau) \mathrm{e}^{-T / \tau}-2(T / \tau)^{2} \mathrm{e}^{-T / \tau}\right] .
\end{aligned}
$$

Eq (14) shows that $\hat{e}$ is a complex function of $T / \tau$. As mentioned above, there are two assumptions of relating $\tau$ to $T$ : (1) $\tau \fallingdotseq T$ (from a conventional viewpoint); and (2) $\tau \ll T$, which is from the slip-pulse concept proposed by Heaton (1990). As $T / \tau \fallingdotseq 1$, the value of $\hat{e}$ from Eq (14) is negative. This is unreasonable, thus, meaning that Model 2 cannot work for the present problem.

\section{Discussion}

In Model 1, a time function of the average displacements, with a $\omega^{-2}$ source spectrum, across a fault plane as proposed by Beresnev and Atkinson (1997) is applied. Results show that as $\tau \fallingdotseq T$ from the conventional viewpoint, $\lg (\hat{e}) \sim-M_{\mathrm{s}}$; while as $\tau \ll T$ from the slip-pulse concept, $\lg (\hat{e}) \sim-(1 / 2) M_{\mathrm{s}}$. The results of Model 1 suggest that as the extreme state of $E_{\mathrm{s}} / M_{0}$ is not taken into account, the source model, including the scaling law and the relation between $\tau$ and $T$, is a significant factor in controlling the correlation of $\hat{e}$ versus $M_{\mathrm{s}}$.

In Model 2, a time function of the average displacements, with a $\omega^{-3}$ source spectrum, across a fault plane as proposed by Beresnev and Atkinson (1997) is applied. Results show that as $\tau \fallingdotseq T$, the value of scaled energy is negative. This means that this model is not acceptable for studying the present problem.

Kanamori and Rivera (2004) modified the $M_{0}-f_{\mathrm{c}}$ correlation from $M_{0} \sim f_{\mathrm{c}}^{-3}$ to $M_{0} \sim f_{\mathrm{c}}^{-(3+\varepsilon)}$, with $0<\varepsilon \leq 1$, 
to yield a positive correction of $\hat{e}$ versus $M_{\mathrm{s}}$. It is interesting to ask if $M_{0}$ can relate to $f_{\mathrm{c}}$ in a form of $M_{0} \sim f_{\mathrm{c}}^{-3.5}$ or not. One of the major factors in controlling the generation of events is the ratio of the fault width, $W$, to the characteristic width, $W_{\mathrm{s}}$, of the seismogenic zone. Small events happen when $W / W_{\mathrm{s}}<1$ and large ones do when $W / W_{\mathrm{s}}$ is equal to or slightly larger than 1 . The $W_{\mathrm{s}}$ could change from area to area. From $M_{0}=\mu \delta L W$, we can examine the scaling of $M_{0}$ versus $L$ for different events. For larger events with $L>10 \mathrm{~km}$, Wang and Ou (1998) stated that $M_{0} \sim L^{2}$ due to $\delta \sim L$ and $\mathrm{W} \fallingdotseq$ constant. Although they did not clearly report the scaling for smaller events with $L<10 \mathrm{~km}$, from their figures, it can be seen that $M_{0} \sim L^{3}$ due to $\delta \sim L$ and $\mathrm{W} \sim L$. For both $\mathrm{P}$ - and $\mathrm{S}$-waves, $f_{\mathrm{c}}$ is inversely proportional to the dimension of the earthquake source, i.e., $f_{\mathrm{c}} \sim L^{-1}$, (cf. Aki and Richards 1980), and, thus, $M_{0} \sim f_{\mathrm{c}}^{-2}$ for large earthquake and $M_{0} \sim f_{\mathrm{c}}^{-3}$ for small events. Obviously, the exponents of the $M_{0}-f_{\mathrm{c}}$ scaling are -2 and -3 , which are both not larger than 3 , for large and small earthquakes, respectively.

\section{Conclusions}

The correlation of $\hat{e}$ versus $M_{\mathrm{s}}$ is studied using two models: (1) the first one is the application of the time function of the average displacements, with an $\omega^{-2}$ spectrum, across a fault plane; and (2) the second one is the application of the time function of the average displacements, with an $\omega^{-3}$ spectrum, across a fault plane. The two models are proposed by Beresnev and Atkinson (1997). Like Kanamori and Rivera (2004), Model 1 gives independence of $\hat{e}$ on $M_{\mathrm{s}}$. This means that in the extreme state of $E_{\mathrm{s}} / M_{0}$, the two different initial conditions, which associated with different source models, lead to a same conclusion that the scaled energy is of size-independence. For Model 2, the value of scaled energy is negative as $\tau \fallingdotseq T$. The results of this study suggest that the source model, including the scaling law and the relation between $\tau$ and $T$, is a factor, yet not a unique one, in controlling the correlation of $\hat{e}$ versus $M_{\mathrm{s}}$. This will depend upon whether the extreme state of $E_{\mathrm{s}} / M_{0}$ is taken into account or not. At present, it is not yet known which model is the most appropriate one to explain the correlation of $\hat{e}$ versus $M_{\mathrm{s}}$, because the observed correlation is still questionable due to high uncertainties in the estimates of $E_{\mathrm{s}}$, especially for large earthquakes.

Acknowledgments This study was sponsored by Academia Sinica and the National Science Council (under Grant No. NSC101-2119-M001-01.

\section{References}

Aki K (1967) Scaling law of seismic spectrum. J Geophys Res 72:1217-1231

Aki K, Richards PG (1980) Quantitative seismology. W. H. Freeman and Co., San Francisco $932 \mathrm{pp}$

Beresnev IA, Atkinson GM (1997) Modeling finite-fault radiation from the $\omega^{-n}$ spectrum. Bull Seism Soc Am 87:67-84

Brodsky EE, Kanamori H (2001) Elastohydrodynamic lubrication of faults. J Geophys Res 106(B8):16357-16374

Brune J (1970) Tectonic stress and seismic shear waves from earthquakes. J Geophys Res 75:4997-5009

Choy GL, Boatwright JL (1995) Global patterns of radiated seismic energy and apparent stress. J Geophys Res 100(18205-18228):1995

Haskell N (1966) Total energy and energy spectral density of elastic radiation from propagating faults, 2, a statistical model. Bull Seism Soc Am 56:125-140

Heaton T (1990) Evidence for and implications of self-healing pulses of slip in earthquake rupture. Phys Earth Planet Phys 64:1-20

Huang MW, Wang JH (2009) Scaled energies of $M \geqq 5.1$ aftershocks of the 1999 Chi-Chi, Taiwan, earthquake estimated from local seismograms. Terr Atmos Ocean Sci 20(5):671-685

Huang MW, Wang JH, Hwang RD, Chen KC (2002) Estimates of two larger-sized aftershocks of the 1999 Chi-Chi (Taiwan) earthquake in the Chia-Yi area. Terr Atmo Ocean Sci 13:299-312

Hwang RD, Wang JH, Huang BS, Chen KC, Huang WG, Chang TM, Chiu HC, Tsai CC (2001) Estimates of stress drop of the ChiChi, Taiwan, earthquake of September 20, 1999 from near-field seismograms. Bull Seism Soc Am 91:1158-1166

Ide S, Beroza GC (2001) Does apparent stress vary with earthquake size? Geophys Res Lett 28:3349-3352

Kanamori H (1977) The energy release in great earthquakes. J Geophys Res 82:2981-2987

Kanamori H, Anderson D (1975) Theoretical basis of some empirical relations in seismology. Bull Seism Soc Am 65:1073-1095

Kanamori H, Heaton TH (2000) Microscopic and macroscopic physics of earthquakes. In: Rundle JB, Turcotte DL, Klein W (eds) Geocomplexity and the physics of earthquakes, AGU geophys. Mono, 120th edn. AGU, Washington, D.C, pp 147-164

Kanamori H, Rivera L (2004) Static and dynamic scaling relations for earthquakes and their implications for rupture speed and stress drop. Bull Seism Soc Am 94:314-319

Kikuchi M, Fukao Y (1988) Seismic wave energy inferred from longperiod body wave inversion. Bull Seism Soc Am 78:1707-1724

Kinoshita S, Ohike M (2002) Scaling relations of earthquakes that occurred in the upper part of the Philippine sea plate beneath the Kanto region, Japan, estimated by means of borehole recordings. Bull Seism Soc Am 92:611-624

Purcaru G, Berckhemer H (1978) A magnitude scale for very large earthquakes. Tectonophys 49:189-198

Shaw BE (1998) Far-field radiated energy scaling in elastodynamic earthquake fault models. Bull Seism Soc Am 88:1457-1465

Vassiliou MS, Kanamori H (1982) The energy release in earthquakes. Bull Seism Soc Am 72:371-387

Wang JH (2004) The seismic efficiency of the 1999 Chi-Chi, Taiwan, earthquake. Geophys Res Lett 31:L10613. doi:10.1029/204GL019417

Wang JH, Ou SS (1998) On scaling of earthquake faults. Bull Seism Soc Am 88:758-766 